\title{
Cilostazol Improves Hyperglycemia-Induced Impairment of Angiogenesis by Stimulating Adiponectin/Adiponectin Receptor1/Sirtuin1
}

\section{Shih-Ya Tseng}

National Cheng Kung University Hospital

Hsien-Yuan Chang

National Cheng Kung University Hospital

Yi-Heng Li

National Cheng Kung University Hospital

Ting-Hsing Chao ( $\sim$ chaotinghsing@gmail.com )

National Cheng Kung University Hospital

\section{Research Article}

Keywords: Cilostazol, angiogenesis, adiponectin, Sirtuin, adiponectin receptor, hyperglycemia.

Posted Date: November 17th, 2021

DOI: https://doi.org/10.21203/rs.3.rs-1038078/v1

License: (c) (i) This work is licensed under a Creative Commons Attribution 4.0 International License. Read Full License 

stimulating adiponectin/adiponectin receptor1/Sirtuin1

*Shih-Ya Tseng ${ }^{1,2},{ }^{*}$ Hsien-Yuan Chang ${ }^{1,3}$, Yi-Heng Li $^{1}$, Ting-Hsing Chao ${ }^{1,4}$

4 1. Division of Cardiology, Department of Internal Medicine, National Cheng Kung

5 University Hospital, College of Medicine, National Cheng Kung University,

6 Tainan, Taiwan

7 2. Department of Biological Science, National Sun Yat-Sen University, Kaohsiung,

8 Taiwan

9 3. Institute of Clinical Medicine, College of Medicine, National Cheng Kung

10 University, Tainan, Taiwan

11 4. Health Management Center, National Cheng Kung University Hospital, Tainan,

12 Taiwan

$13 *$ Both authors contributed equally to this work.

14 Corresponding author:

15 Ting-Hsing Chao, MD

16 Division of Cardiology, Department of Internal Medicine, National Cheng Kung

17 University Hospital

18138 Sheng-Li Road North District, Tainan 70403, Taiwan

19 TEL: +886-6-23523535, ext. 2392 
$1 \quad$ FAX: +886-6-2753834

2 E-mail: chaotinghsing@gmail.com or chaoth@ mail.ncku.edu.tw

3 Running title: Cilostazol improves angiogenesis by increasing adiponectin.

4 


\section{ABSTRACT}

2 Background: Cilostazol is an antiplatelet agent with vasodilating effects that

3 functions by increasing the intracellular concentration of cyclic adenosine

4 monophosphate. However, the effect of cilostazol on adiponectin is still unclear.

5 Purpose: We investigated the effects of cilostazol on adiponectin/adiponectin

6 receptors and the Sirtuin 1 (SIRT1)/AMP-activated protein kinase (AMPK) signaling

7 pathway to prevent high glucose (HG)-induced impairment of angiogenesis in vitro

8 and in vivo.

9 Methods and Results: Human umbilical vein endothelial cells (HUVECs) and human aortic smooth muscle cells (HASMCs) were cocultured in HG conditions.

11 Adiponectin concentrations in the supernatant were significantly increased when HASMCs were treated with cilostazol but not significantly changed when only HUVECs were treated with cilostazol. Cilostazol treatment restored the expression of the adipoR1 and SIRT1 proteins and upregulated the phosphorylation of AMPK $\alpha 1$ in the HUVECs treated with HG but not adipoR2. Cilostazol prevented apoptosis and stimulated proliferation, chemotactic motility and capillary-like tube formation in

17 HG-treated HUVECs through the adipoR1/AMPK/SIRT1 signaling pathway. In

18 cilostazol-treated mice, recovery of the blood flow ratio after hindlimb ischemia and

19 circulating $\mathrm{CD} 34^{+} \mathrm{CD} 45^{\mathrm{dim}}$ cells were significantly attenuated by adipoR 1 knockdown 
1 but not adipoR2 knockdown. The expression of SIRT1, phosphorylation of

2 AMPK $\alpha 1 /$ acetyl-CoA carboxylase and Akt/endothelial nitric oxide synthase in

3 ischemic muscles were significantly attenuated by gene knockdown of adipoR1.

4 Conclusions: Cilostazol prevents HG-induced endothelial dysfunction in vascular

5 endothelial cells and enhances angiogenesis in hyperglycemic mice by upregulating

6 the expression of adiponectin/adipoR1 and its SIRT1/AMPK downstream signaling

7 pathway.

8

9 Keywords: Cilostazol, angiogenesis, adiponectin, Sirtuin, adiponectin receptor,

10 hyperglycemia. 
1

2

3 indicated for peripheral artery disease patients suffering from intermittent

4 claudication. $^{1,2}$ This drug increases cyclic adenosine monophosphate (cAMP) levels

5 by inhibiting PDE and has antiplatelet and vasodilatory effects. Cilostazol was also

6 shown to have pleiotropic effects on endothelial cells, including protection from

7 apoptotic cell death, ${ }^{3}$ increased endothelial nitric oxide synthase (eNOS) activity, ${ }^{4}$

8 stimulation of the release of angiogenic factors, ${ }^{5}$ and improvement of endothelial

9 function. ${ }^{6}$ In addition, several studies have demonstrated that cilostazol has beneficial

10 effects on endothelial progenitor cells ${ }^{7,8}$ and vascular angiogenic effects in a murine

11 hindlimb ischemia model. ${ }^{5}$ Our study also showed that the vascular angiogenic effects

12 of cilostazol are modulated by adenosine monophosphate-activated protein kinase

13 (AMPK)/acetyl-coenzyme A carboxylase (ACC) and probably the subsequent

14 Akt/eNOS pathway. ${ }^{6}$ Therefore, AMPK plays a vital role in the effect of cilostazol

15 and angiogenesis.

16

17 and has beneficial effects on insulin sensitivity, lipid metabolism, and vasoprotective

18 properties, ${ }^{10,11}$ which are mainly mediated by the receptors adipoR 1 , adipoR2, and T-

19 cadherin. AdipoR1 is abundantly expressed in skeletal muscle and is associated with 
1 activation of the AMPK pathway, ${ }^{12,13}$ whereas adipoR2 is abundantly expressed in the

2 liver and is associated with peroxisome proliferator-activated receptor alpha (PPAR $\alpha$ )

3 ligand activity. ${ }^{14,15}$ Several clinical studies have indicated that hypoadiponectinemia is

4 associated with endothelial dysfunction, ${ }^{16-18}$ and animal studies have also shown that

5 adiponectin has anti-atherosclerosis ${ }^{19,20}$ and angiogenesis ${ }^{21}$ effect. In addition,

6 previous studies showed that the effect of adiponectin was mediated by the

7 AMPK/sirtuin-1 (SIRT) pathway. ${ }^{22-24}$ Thus, by activating the AMPK/SIRT axis,

8 adiponectin also plays a vital role in angiogenesis. Notably, adiponectin is not only

9 produced by adipose tissue but also derived from vascular smooth muscle cells

$10(\mathrm{SMCs})^{25}$ and plays a critical function in the contractile phenotype. ${ }^{26}$ Only one

11 clinical study showed that cilostazol increases adiponectin levels, ${ }^{27}$ and the

12 mechanism is unclear. However, to the best of our knowledge, no study has evaluated

13 the effect of cilostazol on adiponectin in terms of angiogenesis and its mechanism.

14 Herein, we hypothesized that cilostazol prevents endothelial dysfunction in

15 vascular endothelial cells and enhances angiogenesis by upregulating adiponectin

16 expression. Therefore, we conducted this study to investigate the effects of cilostazol

17 on adiponectin/adiponectin receptors and the AMPK/SIRT1 signaling pathway to

18 prevent high glucose (HG)-induced impairment of angiogenesis in vitro and in vivo.

\section{Materials and Methods}




\section{Reagents}

2

3 2-phenylindole), TRIzol ${ }^{\circledR}$ reagent and trypsin solution were purchased from

4 Invitrogen (CA, U.S.A.). Cilostazol, EX527 (SIRT1 inhibitor), Giemsa, Griess-

5 Romijn, crystal violet reagent, dimethyl sulfoxide, and the anti- $\beta$-actin antibody were

6 purchased from Sigma-Aldrich (MO, U.S.A.). SRT 1720 (SIRT1 activator) was

7 purchased from Cayman Chemical (MI, U.S.A.). The polymerase chain reaction

8 (PCR) primers for adiponectin and GAPDH were purchased from Pollster

9 Biotechnology (Taipei, Taiwan). An antibody against adiponectin (\#21613-1) was

10 purchased from Proteintech (IL, U.S.A.). Antibodies against phosphorylated Akt

11 (Ser437, \#9271), Akt, phosphorylated AMPK- $\alpha 1$ (Thr172, \#2535), AMPKa1 (\#2603),

12 phosphorylated ACC (Ser79, \#3661), ACC (\#3676) and SIRT1 (\#9475) were

13 purchased from Cell Signaling Technology (MA, USA). Antibodies against

14 phosphorylated eNOS (Ser1177, \#612393) and eNOS (\#610297) were purchased from and adipoR2 (\# sc-46751) were purchased from Santa Cruz Biotechnology (Santa

17 Cruz Biotechnology, TX, U.S.A.). Matrigel and rat monoclonal antibodies against

18 murine CD31 (\#557355) were purchased from BD Biosciences (CA, U.S.A.).

BD Transduction Laboratories (CA, U.S.A.). Antibodies against adipoR1 (\# sc-46749)$$
\text { murine CD31 ( } \$ 557355) \text { were purchased from BD Biosciences (CA, U.S.A.). }
$$

Biotinylated rabbit anti-rat secondary antibodies, AECs (3-amino-9-ethylcarbazole) 
1 and streptavidin-HRP (horseradish peroxidase) were purchased from Dako (Glostrup,

2 Denmark). A BrdU (5-bromo-2-deoxyuridine) kit was purchased from Roche

3 Diagnostics (Mannheim, Germany).

\section{Cell culture}

Human umbilical vein endothelial cells (HUVECs) were freshly isolated from

6 human umbilical cord veins of newborn babies with previously described methods. ${ }^{28}$

7 The present study was performed in accordance with the approved guidelines of the

8 Declaration of Helsinki. Informed consent was obtained from all the participating

9 mothers prior to sample collect. Each patient provided written informed consent. The study protocol was approved by the Institutional ethics committees of National Cheng

11 Kung University Hospital (IRB number: ER-100-072). Cells were cultured in medium 199 containing $20 \%$ FBS, $25 \mathrm{pg} / \mathrm{ml}$ endothelial cell growth factor, 10 units $/ \mathrm{ml}$

13 heparin, and 100 units $/ \mathrm{ml}$ penicillin at $37^{\circ} \mathrm{C}$ in a $5 \% \mathrm{CO}_{2}$ and $95 \%$ humidity

14 incubator. Cells cultured up to the third passage were used in all experiments. Primary passage cryopreserved human aortic smooth muscle cells (HASMCs) were obtained from Cascade Biologics (OR, U.S.A.). Cells were plated in 75-cm2

17 flasks and incubated in M199 supplemented with 10\% FBS and 5\% smooth muscle

18 growth supplement (SMGS). Cells cultured up to passages 6 to 9 were used for experiments. 
2 HG conditions.

\section{Transwell coculture experiments}

$4 \quad$ Coculture of cells was performed using a Transwell system. Transwell chambers

$5 \quad(0.4 \mu \mathrm{m}$ pore diameter, Costar) were used to establish HUVEC and HASMC coculture

6 models. HASMCs were seeded at a density of $2.5 \times 10^{3}$ cells $/ \mathrm{cm}^{2}$ in the upper

7 chamber, and HUVECs were seeded at a density of $1 \times 10^{4}$ cells $/ \mathrm{cm}^{2}$ in the lower

8 chamber. The upper and lower compartments were separated by a polycarbonate

9 membrane to allow the free circulation of various cytokines and metabolites secreted

10 by the cells. For preparation of conditioned medium (CM), HASMCs were plated in

11 6-well dishes and grown until 90\% confluence. Afterwards, the culture medium was

12 incubated in M199 medium with 1\% FBS, HG, and $100 \mu \mathrm{M}$ cilostazol for 2 days

13 (HASMC-CM).

14 Immunofluorescence staining

15 Transwell membranes were fixed in 4\% paraformaldehyde and permeabilized

16 with $0.2 \%$ Triton X-100. The cells were then incubated overnight with primary Cys-

17 anti- $\alpha$ SMA antibody (Sigma, U.S.A.) for SMCs and/or primary anti-CD31 antibody

18 (Dako, Denmark) for epithelial cells. After the cells were washed with $0.1 \%$ Tween in

19 PBS, they were incubated for 30 min with secondary Alexa Fluor 488-goat anti-rabbit 
1 antibody (Invitrogen, U.S.A.). Nuclei were labeled with DAPI.

2 For ischemic muscle staining, samples were fixed with paraformaldehyde and

3 embedded in paraffin. DAPI was used to label cell nuclei, and a primary anti-

4 adiponectin antibody (Proteintech, IL, U.S.A.) was used to label adiponectin.

$5 \quad$ Enzyme-linked immunosorbent assay (ELISA)

6

Adiponectin levels in the supernatants of HASMCs and HUVECs were measured

7 by ELISAs using a commercial ELISA kit (R\&D Systems,IL, U.S.A.), as described

8 by the manufacturer.

9 Short hairpin RNA (shRNA) transfection

HUVECs were transfected with 1-2 $\mu$ g plasmid DNA with Opti-MEM I medium

11 and Lipofectamine 3000 (Invitrogen) reagent for $15 \mathrm{~min}$, according to the

manufacturer's instructions. All plasmids were purchased from the National RNAi

13 Core Facility (Taipei, Taiwan). After transfection for $48 \mathrm{~h}$, the cells were treated with

14 various reagents in serum-free M199 medium. The oligonucleotide sequences of the

15 sRNAs used are as follows:

16 AdipoR1 (5'-CCACTTCTATGGAGTCTCCAA-3');

17 AdipoR2 (5'- GCTCTTCTCTAAACTGGATTA -3');

18 AMPK $\alpha 1$ (5'CCTGGAAGTCACACAATAGAA-3');

SIRT1 (5'-CAGGTCAAGGGATGGTATTTA-3'); 
1 with nontarget RNA as a control.

\section{Proliferation assay}

BrdU incorporation was analyzed using a cell proliferation ELISA kit (Roche

4 Diagnostics, Mannheim, Germany) according to the manufacturer's protocol.

\section{Migration assay}

The migration of HUVECs was tested with $8.0 \mu \mathrm{m}$ Transwell inserts (Corning,

7 MA, USA). HUVECs $\left(1 \times 10^{5}\right.$ cells in $\left.100 \mu \mathrm{L}\right)$ were placed in the upper chamber with

8 M199 medium supplemented with 0.5\% serum, and HASMCs with or without

9 cilostazol were placed in the lower chamber. After incubation at $37^{\circ} \mathrm{C}$ for $4 \mathrm{~h}$, the cells were fixed with 4\% paraformaldehyde and stained with Giemsa solution. Migrated

11 cells were calculated using an Olympus CKX31 microscope (Tokyo, Japan) with Image-Pro Plus software (NIH, Littleton, CO, USA).

\section{Matrigel tube formation}

HUVECs $\left(2 \times 10^{4}\right.$ cells/well $)$ were plated on top of $200 \mu \mathrm{L}$ of Matrigel Matrix relative tube length obtained from image analysis using MacBiophotonics ImageJ

17 software.

\section{Analysis of apoptosis}


1 (Roche Diagnostics, Germany) according to the manufacturer's protocol.

2 Measurement of Nitric Oxide production

Supernatants of HUVECs were collected after coculture with HASMCs for $1 \mathrm{~h}$.

4 One hundred microliters of supernatant was mixed with $10 \mathrm{~mL}$ of Griess-Romijn

5 reagent $(100 \mathrm{mg} / \mathrm{mL})$. The absorbance of the mixtures was spectrophotometrically

6 measured at a wavelength of $540 \mathrm{~nm}$.

7 RNA isolation, reverse transcription, and real-time polymerase chain reaction

8 (PCR)

Total RNA was extracted using TRIzol reagent (Invitrogen, U.S.A.) according to

10

11

12

13

14

15

16

17

18

19

the manufacturer's protocol and then reverse transcribed into cDNA using

MultiScribe $^{\mathrm{TM}}$ Reverse Transcriptase (Applied Biosystems, MA, U.S.A.). Gene

expression was quantitated using SYBR Green (Qiagen, CA, U.S.A.) in a Step One

Plus real-time PCR system (Applied Biosystems, U.S.A.). The expression levels of each mRNA were calculated by the $2^{-\Delta \Delta \mathrm{Ct}}$ method, and GAPDH was used as an

internal control. The respective primers were as follows: adiponectin (forward: 5'-

ACAGGAGATGTTGGAATGACAG-3'; reverse: 5'-CTGC

CGTCATAATGATTCTGTT-3'), GAPDH (forward: 5'-

TGGCAACAATATCCACTTTACC-3'; reverse: 5'-AAGGTG

AAGGTCGGAGTCAAC-3'). 


\section{Western blot analysis}

HUVECs were lysed using RIPA lysis buffer (Toolsbiotech, Taipei, Taiwan), and

3 the protein concentration was determined by using a BCA Protein Assay Kit (Thermo

4 Fisher Scientific, U.S.A.). Approximately $30 \mu \mathrm{g}$ of protein samples was separated by

$5 \quad 8-10 \%$ SDS/PAGE (polyacrylamide gel) and then transferred to PVDF membranes

6 (Millipore, MA, U.S.A.). Primary antibodies against the following targets were used:

7 adipoR1, adipoR2, SIRT1, phosphorylated AMPK $\alpha 1$ (Thr172), AMPK- $\alpha 1$,

8 phosphorylated ACC (Ser79), ACC, phosphorylated eNOS (Ser1177 and Thr495),

9 eNOS, phosphorylated Akt (Ser473), Akt and $\beta$-actin. The membranes were exposed

10 to HRP-conjugated secondary antibodies, and the blots were developed by enhanced

11 chemiluminescence (PerkinElmer, CT, USA), followed by exposure to X-ray film

12 (Fujifilm Medical, Tokyo, Japan). $\beta$-actin was used as an internal control.

13 Hyperglycemic mice and murine hindlimb ischemia model

These animal experiments were performed at the College of Medicine, National Cheng Kung University (Tainan, Taiwan). All experiments performed on the animals were approved by the institutional Animal Care and Use Committee, National Cheng

17 Kung University, Tainan, Taiwan (IACUC number: 100024). All experiments are

18 conducted in accordance with relevant institutional and ARRIVE ${ }^{29}$ guidelines and

19 regulations. Eight-week-old male ICR mice were intraperitoneally injected with 
1 streptozotocin (STZ; $150 \mathrm{mg} / \mathrm{kg}$ ) in citrate buffer to induce experimental

2 hyperglycemia. After 2 weeks, the plasma glucose level after overnight fasting was

3 measured to confirm that these mice were hyperglycemic.

$4 \quad$ Hyperglycemic mice were anesthetized with an intraperitoneal injection of

5 pentobarbital $(80 \mathrm{mg} / \mathrm{kg})$, followed by ligation of the proximal segment of the right

6 femoral artery as previously described. ${ }^{5,30}$ Cilostazol (10 mg/kg of body weight) was

7 injected intraperitoneally 30 min prior to induction of ischemia and then twice a day

8 at the same dose from days 2 to 7 . Control animals received equivalent volumes of

9 normal saline. The mice were sacrificed by inhalation of excessive carbon dioxide 28

10 days after the surgery.

\section{Knockdown of adipoR1 and adipoR2 in the hindlimb}

The constructs of sh-adipoR1, sh-adipoR 2 and sh-pLk0.1 were provided with

$13 \quad 10 \%$ glucose solution. Plasmid and in vivo jetPEI reagent were dissolved and prepared

14 according to the optimized manufacturer's protocols $(\mathrm{N}: \mathrm{P}=10)$. One hundred

15 microliters of transfection complexes was injected intramuscularly into multiple sites

16 of hindlimbs on days $0,7,14$, and 21 after inducing hindlimb ischemia.

\section{Laser Doppler flow imaging}

Blood perfusion in hindlimb blood flow was measured using a laser Doppler

perfusion imaging analyzer (Moor Instruments, Devon, UK) preoperatively and 
1 postoperatively on days $3,7,14,21$ and 28 after surgery. The digital color-coded

2 images were analyzed to quantify the blood flow in the region from the knee joint to

3 the toe. The perfusion rate (\%) was calculated as the ratio of blood flow on the

4 ipsilateral site compared with the contralateral side.

5 Measurement of capillary density in ischemic limbs

6 Muscle tissue from the ischemic gastrocnemius muscle was harvested, fixed in

7 methanol, paraffin-embedded, and cross-sectioned for histological immunostaining.

8 Primary antibody against CD31 (1:50) for capillary detection was incubated at $4{ }^{\circ} \mathrm{C}$

9 overnight. Sections were then incubated with biotinylated rabbit anti-rat secondary antibody (1:500) for $30 \mathrm{~min}$. Streptavidin-HRP was added for $10 \mathrm{~min}$, and color

11 development was performed by the addition of the AEC substrate. Five sections were randomly studied for each mouse. The slides were counterstained with hematoxylin,

13 and digital images were captured using an Olympus IX71 microscope. Capillary

14 density was quantified by counting the mean number of capillaries, as revealed by

15 positive expression of CD31 on endothelial cells, and capillary/fiber ratio, as the ratio

16 of the number of CD31-positive endothelial cells to the number of myofibers.

\section{Enumeration of circulating progenitor cells}


1 (ISHAGE) guidelines. Briefly, $50 \mu \mathrm{L}$ of whole blood obtained from mouse tail veins

2 was incubated with phycoerythrin-conjugated anti-CD34 and FITC-conjugated anti-

3 CD45 monoclonal antibodies (BD Pharmingen, U.S.A.) in a TruCOUNT tube (BD

4 Pharmingen, U.S.A) following the manufacturer's instructions. CD34+CD45dim cells

5 were then analyzed and quantified on a FACSCanto flow cytometer according to the

6 ISHAGE sequential gating strategy.

\section{$7 \quad$ Statistical analysis}

8 All data are expressed as the mean \pm standard error of the mean (SEM), and each

9 point represents the average of at least 3 separate experiments. Statistical analysis was performed using Student's t-test to compare the statistical significance of multiple

11 groups. Statistical significance was tested at $P<0.05$.

13 Results production from HASMCs. After seeding and incubation of HASMCs, HUVECs were cocultured under HG conditions and then treated with cilostazol (Figure 1A).

17 Both HASMCs and HUVECs were confirmed by immunofluorescence (Figure 1B).

18 Under HG conditions, the concentrations of adiponectin in the SMC supernatants

19 were significantly decreased compared with those in the mannitol group without HG 
$1 \quad(2.29 \pm 0.12$ vs. $3.33 \pm 0.35 \mathrm{ng} / \mathrm{mL})$. These results indicated attenuated secretion of

2 adiponectin from HASMCs under HG conditions. However, after treatment with 100

$3 \mu \mathrm{M}$ cilostazol under HG conditions, the adiponectin level was significantly increased

$4 \quad(3.64 \pm 0.27$ vs. $2.29 \pm 0.12 \mathrm{ng} / \mathrm{mL}$, Figure 1C-D). This finding implied that cilostazol

5 reverses the HG-induced reduction in adiponectin secretion from HASMCs. In

6 addition, the adiponectin concentrations in the HUVEC supernatants were

7 significantly increased in a dose-dependent manner when HASMCs were treated with

8 cilostazol but not significantly changed when only HUVECs were treated with

9 cilostazol (Figure 1E). Accordingly, cilostazol increased the adiponectin

10 concentrations in the supernatant of the Transwell cultured with HUVECs via

11 paracrine effects from SMCs.

13 adiponectin receptors and enhancing adiponectin released from SMCs by

14 binding to adiponectin receptors in HUVECs. To investigate how HUVECs were

15 affected after coculture with HASMCs treated with cilostazol, we transfected sh-

16 adipoR1 and sh-adipoR2 to knock down adiponectin receptors in HUVECs. Our data

17 showed that cilostazol significantly stimulated cell proliferation $(1.38 \pm 0.08$ vs. 1.08

$18 \pm 0.08)$ and migration $(81.8 \pm 12.8$ vs. $59.6 \pm 9.3$ cells/field $)$ in the HG-treated

19 HUVECs, and these effects of cilostazol were attenuated after knockdown of the 
1 adiponectin receptors, especially adipoR1 (Figure 2A-B). With regard to vascular tube

2 formation, the HG-treated HUVECs failed to form networks, but the capillary-like

3 tube network was more enriched and longer when HUVECs were treated with

4 cilostazol (relative tube length: $76.8 \pm 12.8$ vs. $55.3 \pm 8.6 \mu \mathrm{m} /$ field). However, after

5 knockdown of adipoR1, the tube length was significantly shortened (45.6 $\pm 4.9 \mathrm{vs.}$

$676.8 \pm 12.8 \mu \mathrm{m} /$ field, Figure 2C). A similar antiapoptotic effect was also found

7 (Figure 2D). In addition, after knockdown of adiponectin receptors, the stimulatory

8 effect of cilostazol on the expression of the downstream signaling molecules $p$ -

9 AMPK $\alpha 1$ and SIRT1 was also decreased (Figure 2E). Thus, adiponectin receptors, especially adipoR1, play an important role in the angiogenic effect of cilostazol by

11 increasing adiponectin secretion from HASMCs. the expression of the downstream pathway components AMPK/SIRT in the HUVECs cocultured with the cilostazol-treated HASMCs. As expected, HG conditions decreased the phosphorylation of AMPK $\alpha 1$ and SIRT1; however, cilostazol treatment restored the phosphorylation of both molecules (Figure 3A). When AMPK $\alpha 1$ was

17 knocked down, the stimulating effect of cilostazol on the SIRT1 protein was

18 diminished (Figure 3B-C). However, the phosphorylation of AMPK was significantly decreased $(0.43 \pm 0.08$ vs. $0.62 \pm 0.11)$ after SIRT knockdown, even when HUVECs 
1 were cocultured with cilostazol-treated HASMCs (Figure 3D-E). Furthermore, dual

2 knockdown attenuated the stimulating effect of cilostazol on cell proliferation and

3 migration in the HG-treated HUVECs (Figure 3F-G). This finding indicates that the

4 AMPK/SIRT axis plays an important role in cilostazol-induced angiogenesis. the downstream pathway of adiponectin secreted from cilostazol-treated HASMCs,

7 we used a SIRT enhancer, SRT 1720, and its inhibitor, EX527, for the subsequent

8 experiments. The results showed that cilostazol increased the phosphorylation of ACC

$9 \quad(0.59 \pm 0.05$ vs. $0.37 \pm 0.03)$ and eNOS $(0.25 \pm 0.05$ vs. $0.13 \pm 0.03)$, while EX527 attenuated this effect (Figure 4A-B). In contrast, after treatment with SRT 1720, the

11 phosphorylation of ACC and eNOS was enhanced (p-ACC: $0.60 \pm 0.07$ vs. $0.37 \pm$ 0.03; p-eNOs: $0.46 \pm 0.07$ vs. $0.13 \pm 0.03$, Figure 4A-B). Furthermore, the effect of

13 cilostazol on cell proliferation, migration, and nitric oxide production was decreased by EX527, whereas SRT1720, which served as a positive control, had similar effects to cilostazol (Figure 4C-E). This result demonstrated that the vascular angiogenic effects of cilostazol are modulated by SIRT and its downstream signaling pathway, the

17 ACC/eNOS axis. used STZ-induced diabetic mice treated with cilostazol after hindlimb ischemia to 
1 investigate the effects of cilostazol and adiponectin receptors in angiogenesis. After

2 adiponectin receptor knockdown, the body weights of the mice were not different, but

3 the fasting blood glucose was significantly higher than that of the control group 14-21

4 days after surgery (Day 14: $339 \pm 15$ vs. $307 \pm 14$; Day 21: $353 \pm 15$ vs. $271 \pm 12$

$5 \mathrm{mg} / \mathrm{dL}$, Figure 5A-B). Western blot analysis confirmed that the adiponectin receptor

6 was successfully knocked down (Figure 5C-D). Immunofluorescence staining of

7 ischemic limb muscle showed that the expression of adiponectin increased in the

8 cilostazol group but significantly decreased in the sh-AdipoR1 group $(13.38 \pm 4.52$

9 vs. $32.33 \pm 5.45$ cells, Figure 5E). This finding implied that adipoR1, in turn, was involved in cilostazol-mediated adiponectin secretion. Furthermore, in the sh-adipoR1

11 group, the recovery of the blood flow ratio measured by the ipsilateral limb versus the contralateral limb was significantly lower than that in the other groups $21-28$ days

13 after surgery (Day 21: $0.24 \pm 0.04$ vs. $0.45 \pm 0.04$; Day 28: $0.18 \pm 0.05$ vs. $0.45 \pm$

140.05 , Figure $6 \mathrm{~A}-\mathrm{B})$. In addition, the levels of $\mathrm{CD}^{31+} \mathrm{CD}^{45 \mathrm{dim}}$ cells, $\mathrm{CD}^{34+} \mathrm{CD}^{45 \mathrm{dim}}$ cells,

15 and relevant signaling molecules of adiponectin, including phosphorylation of

16 AMPK $\alpha 1$, ACC, Akt, eNOS and expression of SIRT1, were decreased in the sh-

17 adipoR1 group (Figure 6C-F), suggesting that the angiogenic effects of cilostazol

18 were mediated by adiponectin receptors, especially adipoR1. 
1

2

3 angiogenic function by increasing adiponectin secretion and activating

4 adipoR1/SIRT1 signaling pathways (Figure 7) both in vitro and in vivo.

5

6

7 direct contact, eNOS-derived nitric oxide, extracellular matrix, extracellular vesicles,

8 microRNAs, and other factors. ${ }^{31}$ According to our study, cilostazol stimulates

9 adiponectin secretion from SMCs but not ECs and enhances angiogenic effects

10

11 through crosstalk between ECs and SMCs via paracrine effects. Adiponectin is

12 produced predominantly by adipose tissue, ${ }^{9}$ but whether cilostazol will also stimulate

13 adiponectin secretion from adipocytes is still unknown.

14

15 plays a key role in modulating the energy-consuming anabolic pathway. ${ }^{32}$ AMPK was reported to be indirectly involved in SIRT1 activation through phosphorylation of

$17 \mathrm{GAPDH},{ }^{33}$ and SIRT1 functions as an upstream regulator of liver kinase B1

18 (LKB1)/AMPK signaling. ${ }^{34}$ Both AMPK and SIRT1 mutually regulate each other's

19 activities, a mechanism of action that is required for adiponectin-mediated 
1 cardiovascular protection. ${ }^{24}$ Our data also showed that the AMPK/SIRT axis is

2 required to promote the proliferation of HUVECs cocultured with cilostazol-treated

3 HASMCs. Therefore, we speculate that cilostazol stimulates adiponectin secretion by

$4 \quad$ SMCs and then interacts with adipoR1 to ameliorate angiogenesis through the

5 AMPK/SIRT axis, ACC, and eNOS.

Our previous data demonstrated that cilostazol improves angiogenesis by

7 modulating AMPK/ACC in parallel with the $\mathrm{cAMP} /$ protein kinase A signaling

8 pathway. ${ }^{6}$ In this study, we further identified the role of adiponectin in these two

9 parallel pathways of cilostazol. In a hindlimb ischemia model of diabetic mice,

10 cilostazol increased adiponectin levels and downstream signaling pathways,

11 AMPK/SIRT, ACC, and eNOS, and then improved angiogenesis. However, after

12 adipoR1 knockdown, all downstream pathways and blood flow ratios were attenuated

13 to be similar to those in mice without cilostazol. We speculated that this pathway

14 mediated by adiponectin plays a dominant and important role in the angiogenic effect

15 of cilostazol.

16 In addition to the angiogenic effect, adiponectin is also a therapeutic target for

17 insulin resistance, type 2 diabetes, and metabolic syndrome. ${ }^{10,35}$ A meta-analysis

18 indicated that type 2 diabetes risk was strongly associated with low levels of

19 adiponectin. ${ }^{36}$ The mechanism by which adiponectin improves glucose metabolism 
1 may be through AMPK, interleukin-6, tumor necrosis factor- $\alpha$, inducible nitric oxide

2 synthase, and PPAR $\alpha .{ }^{35}$ However, adiponectin levels have also been reported to be

3 strongly correlated with lipid profiles in patients with type 2 diabetes. ${ }^{37}$ The possible

4 mechanism is because adiponectin can promote apoA-I/HDL-mediated cholesterol

5 efflux via $\mathrm{ABCA} 1 .{ }^{38}$ Of note, since cilostazol can promote the secretion of

6 adiponectin, other cilostazol applications related to adiponectin may be worth further

7 study.

8 In conclusion, cilostazol prevents high glucose-induced endothelial dysfunction

9 in vascular endothelial cells and enhances angiogenesis in hyperglycemic mice by

10 upregulating the expression of adiponectin/adipoR1 and its downstream signaling

11 molecules AMPK/SIRT1. The results suggest that cilostazol provides therapeutic

12 benefit in the treatment of diabetic patients with ischemic disease. 


\section{$1 \quad$ Abbreviations}

2 ACC: Acetyl-coenzyme A carboxylase

3 AdipoR1: Adiponectin receptor-1

4 AdipoR2: Adiponectin receptor-2

$5 \quad$ AMPK $\alpha$ 1: AMP-activated protein kinase $\alpha 1$

6 cAMP: Cyclic adenosine monophosphate

7 CM: Conditioned medium

8 ELISA: Enzyme-linked immunosorbent assay

9 eNOS: Endothelial nitric oxide synthase

10 FBS: Fetal bovine serum

11 HASMCs: Human aortic smooth muscle cells

12 HG: High glucose

13 HUVECs: Human umbilical vein endothelial cells

14 PPAR $\alpha$ : Peroxisome proliferator-activated receptor alpha

15 shRNA: Short hairpin RNA

16 SIRT1: Sirtuin 1

17 SMGS: Smooth muscle growth supplement

18 


\section{Ethics approval and consent to participate:}

3 the Declaration of Helsinki. The study protocol was approved by the Institutional

4 ethics committees of National Cheng Kung University Hospital (IRB number: ER-

5 100-072). All experiments performed on the animals were approved by the

6 institutional Animal Care and Use Committee, National Cheng Kung University,

7 Tainan, Taiwan (IACUC number: 100024). All methods are reported in accordance

8 with ARRIVE guidelines.

9 Consent for publication: Not applicable

Data Availability Statement: The datasets supporting the conclusions of this article

11 are included within the article and its additional file.

Competing Interests: The authors declare that there are no competing interests associated with the manuscript.

14 Funding: This work was supported by funds from the Ministry of Health and Welfare of Taiwan (MOHW108-TDU-B-211-133003) and Ministry of Science and

16 Technology (MOST 106-2811-B-006 -060; MOST 107-2314-B-006 -076-MY2;

17 MOST 107-2811-B-006 -017; MOST 108-2811-B-006 -522; and MOST 109-2314-B-

18 006-069-MY3). 
1 Conception and design: ST, YL, TC

2 Analysis and interpretation: ST, HC, YL, TC

3 Data collection and verification: ST, TC

$4 \quad$ Writing the article: ST, HC

5 Critical revision of the article: ST, HC, YL, TC

6 Final approval: ST, HC, YL, TC

7 Statistical analysis: ST

8 Obtained funding: ST, TC

9 Overall responsibility: TC

10 Acknowledgements: Not applicable 
2 Figure 1. Cilostazol increased adiponectin levels in HUVECs by stimulating

3 HASMC production under high-glucose conditions. (A) Schematic diagram of the

4 Transwell coculture system. HUVECs were seeded in the upper chamber, and

5 HASMCs were cultured in the lower chamber with high-glucose (HG; $25 \mathrm{mmol} / \mathrm{L})$

6 conditions. (B) Representative images of immunostaining of HUVECs with CD31

7 (green) and HASMCs with $\alpha-$ SMA (red). Scale bar, $25 \mu \mathrm{M}$. (C) Quantification of

8 adiponectin levels in the supernatant of the lower insert by ELISAs. Mannitol was

9 used as an osmolality control. (D) Quantification of adiponectin expression in

10 HASMCs by real-time PCR. (E) Quantification of adiponectin levels in the cilostazol-

11 treated coculture system under HG conditions by ELISAs. The results are represented

12 as the mean \pm S.E.M. from three independent experiments. @ $p<0.05$ versus the

13 normal group; $* p<0.05$ and $* * p<0.01$ versus the vehicle group.

Figure 2. Knockdown of adiponectin receptors attenuated the angiogenic

functions of cilostazol in high-glucose (HG)-treated HUVECs. AdipoR1 shRNA,

17 adipoR2 shRNA, and control shRNA (pLk0.1) lentivirus were used to infect

18 HUVECs. Then, the Transwell coculture system was incubated with high glucose in

19 the presence or absence of cilostazol $(100 \mu \mathrm{M})$ on the underside of the inserts for $24 \mathrm{~h}$. 
1 (A) Quantification of BrdU incorporation. (B) Quantification of migrating cells of

2 HUVECs. (C) Representative photomicrographs $(\times 200)$ of the tube formation assay

3 and quantitation of branching points. (D) Quantification of DNA fragmentation by

4 cell death ELISAs. (E) Effects of adiponectin receptor knockdown on cilostazol-

5 induced AMPK phosphorylation and SIRT1 activity. Representative image of Western

6 blot analysis of cell lysates from HUVECs. The results are represented as the mean \pm

$7 \quad$ S.E.M. from triplicate wells in three experiments. @ $P<0.05$ and @@ $P<0.01$

8 versus the vehicle group; ${ }^{*} P<0.05, * * P<0.01$ and $* * * P<0.001$ versus the sh-

$9 \quad$ pLk0.1 group treated with cilostazol $(100 \mu \mathrm{M})$.

Figure 3. Cilostazol upregulated the AMPK/SIRT axis in HUVECs pretreated

with high glucose (HG). (A) Cilostazol stimulated the phosphorylation of AMPK $\alpha 1$ and SIRT1 activity in a dose-dependent manner, with peak improvement at $100 \mu \mathrm{M}$ cilostazol. (B E) Western blot assay of AMPK $\alpha 1$ and SIRT1 in the HUVECs transfected with AMPK $\alpha 1$ shRNA (B), SIRT1 shRNA (D), or nontargeting pLk0.1 shRNA and then cocultured with HASMCs treated with vehicle or cilostazol. (C \&E)

17 Quantification of Western blot data. (F) Quantification of BrdU incorporation data of HUVECs transfected with shRNA and cocultured with cilostazol-treated HASMCs for 24 hours. (G) Quantification of migrating cells. The results are represented as the 
1 mean \pm S.E.M. from three independent experiments performed in triplicate. @ $P<$

$2 \quad 0.05, @ @ P<0.01$, and @@@ $P<0.01$ versus the sh-pLk001+vehicle group; * $P<$

30.05 and $* * P<0.01$ versus the sh-pLk0.1 group treated with cilostazol $(100 \mu \mathrm{M})$.

4

5

6

7

8

9 detected for ACC, p-ACC (A), eNOS and p-eNOS (B) by Western blot analysis. (C)

Quantification of BrdU incorporation. (D) Quantification of migrating cells. (E) NO

11 production in HUVECs. The results are represented as the mean \pm S.E.M. from three independent experiments performed in triplicate. @ @ $P<0.01$ versus the vehicle group; $* P<0.05$ and $* * P<0.01$ versus the cilostazol $(100 \mu \mathrm{M})$ group.

Fig 5. AdipoR1 plays a vital role in the cilostazol-mediated adiponectin increase

in the ischemic limbs. Streptozotocin (STZ)-induced diabetic mice were transfected

17 with adipoR1 sh-RNA or adipoR2 sh-RNA to knock down adiponectin receptors. Animals were then injected with cilostazol (30 mg/kg/) or vehicle twice daily for one week. (A) Body weight and (B) plasma glucose levels in the adipoR1 or adipoR2 
1 knockdown mice. The protein expression levels of AdipoR1 (C) and AdipoR2 (D)

2 were significantly decreased by plasmid transfection. ** $P<0.05$ versus the control

3 group. (E) Representative images of ischemic limb immunofluorescence staining with

4 adiponectin (red), DAPI (nucleus, blue), and their colocalization (merge). Scale bar =

$550 \mu \mathrm{M}$. The results are represented as the mean \pm S.E.M. from five mice per group.

$6 @ P<0.01$ versus the sh-pLk0.1 + vehicle-treated mice; $* P<0.05$ and $* * * P<$

$7 \quad 0.001$ versus the sh-pLk0.1 + cilostazol-treated mice.

8

9 Fig 6. The beneficial effects of cilostazol in revascularization were attenuated by adiponectin receptor knockdown in a limb ischemia model. (A) Laser Doppler

11 analysis of blood perfusion in the hindlimbs of the mice on days 3, 7, 14, 21 and 28.

12 (B) Quantification of the blood flow ratio. (C) Representative photomicrographs

13 showing CD31 (red) and nuclei (blue) in ischemic muscle after femoral artery ligation

14 for four weeks. Scale bar $=100 \mu \mathrm{M}$. (D) Quantification of CD31-positive cells and

15 the ratio of the number of CD31-positive cells to the number of myofibers. (E)

16 Quantification of circulating $\mathrm{CD}^{34+} \mathrm{CD} 45^{\text {dim }}$ cells in mice analyzed by flow

17 cytometry. (F) Western blot assays and quantification of phosphorylation of

18 AMPK $\alpha 1$, ACC, Akt, eNOS, and SIRT1 activity in muscles. The results are

19 represented as the mean \pm S.E.M. from five mice per group. @ $P<0.05$ and @@ $P$ 
$1<0.01$ versus the vehicle group; $* P<0.05$ and $* * P<0.01$ versus the sh-pLk0.1 group

2 treated with cilostazol $(100 \mu \mathrm{M})$.

3

$4 \quad$ Fig 7. Schematic diagram. The diagram shows the vascular angiogenic effect of

5 cilostazol through stimulation of adiponectin secretion, adiponectin receptor 1 , and

6 the SIRT1 signaling pathway. ACC, acetyl-coenzyme A carboxylase; AdipoR1,

7 adiponectin receptor 1; AMPK, adenosine monophosphate-activated protein kinase;

8 EC, endothelial cell; eNOS, endothelial nitric oxide synthase; SIRT1, Sirtuin 1; SMC;

$9 \quad$ smooth muscle cell.

10 


\section{References}

21 Lugnier, C. Cyclic nucleotide phosphodiesterase (PDE) superfamily: a new 3 target for the development of specific therapeutic agents. Pharmacology \& 4 therapeutics 109, 366-398, doi:10.1016/j.pharmthera.2005.07.003 (2006).

52 Aboyans, V. et al. 2017 ESC Guidelines on the Diagnosis and Treatment of doi:10.1042/cs20110432 (2012).

3 Kim, M. J. et al. Protection from apoptotic cell death by cilostazol, phosphodiesterase type III inhibitor, via cAMP-dependent protein kinase activation. Pharmacological research 54, 261-267, doi:10.1016/j.phrs.2006.05.006 (2006).

$4 \quad$ Omi, H. et al. Cilostazol inhibits high glucose-mediated endothelial-neutrophil adhesion by decreasing adhesion molecule expression via NO production. Microvascular research 68, 119-125, doi:10.1016/j.mvr.2004.05.002 (2004).

5 Chao, T. H. et al. A novel vasculo-angiogenic effect of cilostazol mediated by cross-talk between multiple signalling pathways including the ERK/p38 MAPK signalling transduction cascade. Clin Sci (Lond) 123, 147-159,

Tseng, S. Y. et al. Cilostazol improves high glucose-induced impaired angiogenesis in human endothelial progenitor cells and vascular endothelial cells as well as enhances vasculoangiogenesis in hyperglycemic mice mediated by the adenosine monophosphate-activated protein kinase pathway. J Vasc Surg 63, 1051-1062 e1053, doi:10.1016/j.jvs.2014.10.103 (2016).

7 Kawabe-Yako, R., li, M., Masuo, O., Asahara, T. \& Itakura, T. Cilostazol activates function of bone marrow-derived endothelial progenitor cell for reendothelialization in a carotid balloon injury model. PloS one 6, e24646, doi:10.1371/journal.pone.0024646 (2011).

Lee, D. H. et al. Cilostazol enhances integrin-dependent homing of progenitor cells by activation of cAMP-dependent protein kinase in synergy with Epac1. Journal of neuroscience research 89, 650-660, doi:10.1002/jnr.22558 (2011). 9 Maeda, K. et al. cDNA cloning and expression of a novel adipose specific 
collagen-like factor, apM1 (AdiPose Most abundant Gene transcript 1). Biochemical and biophysical research communications 221, 286-289, doi:10.1006/bbrc.1996.0587 (1996).

10 Kadowaki, T. et al. Adiponectin and adiponectin receptors in insulin resistance, diabetes, and the metabolic syndrome. J Clin Invest 116, 17841792, doi:10.1172/jci29126 (2006).

11 Zhu, W., Cheng, K. K., Vanhoutte, P. M., Lam, K. S. \& Xu, A. Vascular effects of adiponectin: molecular mechanisms and potential therapeutic intervention. Clin Sci (Lond) 114, 361-374, doi:10.1042/CS20070347 (2008).

12 Yamauchi, T. et al. Adiponectin stimulates glucose utilization and fatty-acid oxidation by activating AMP-activated protein kinase. Nature medicine $\mathbf{8}$, 1288-1295, doi:10.1038/nm788 (2002).

13 Tomas, E. et al. Enhanced muscle fat oxidation and glucose transport by ACRP30 globular domain: acetyl-CoA carboxylase inhibition and AMPactivated protein kinase activation. Proceedings of the National Academy of Sciences of the United States of America 99, 16309-16313, doi:10.1073/pnas.222657499 (2002).

14 Yamauchi, T. et al. Cloning of adiponectin receptors that mediate antidiabetic metabolic effects. Nature 423, 762-769, doi:10.1038/nature01705 (2003).

15 Yamauchi, T. et al. Globular adiponectin protected ob/ob mice from diabetes and ApoE-deficient mice from atherosclerosis. The Journal of biological chemistry 278, 2461-2468, doi:10.1074/jbc.M209033200 (2003).

16 Shimabukuro, M. et al. Hypoadiponectinemia is closely linked to endothelial dysfunction in man. The Journal of clinical endocrinology and metabolism 88, 3236-3240, doi:10.1210/jc.2002-021883 (2003).

17 Torigoe, M. et al. Impact of the high-molecular-weight form of adiponectin on endothelial function in healthy young men. Clinical endocrinology 67, 276281, doi:10.1111/j.1365-2265.2007.02876.x (2007).

18 Okui, H. et al. Adiponectin is a better predictor of endothelial function of the coronary artery than HOMA-R, body mass index, immunoreactive insulin, or triglycerides. International journal of cardiology 126, 53-61, doi:10.1016/j.ijcard.2007.03.116 (2008).

19 Okamoto, Y. et al. Adiponectin reduces atherosclerosis in apolipoprotein Edeficient mice. Circulation 106, 2767-2770, doi:10.1161/01.cir.0000042707.50032.19 (2002).

20 Matsuda, M. et al. Role of adiponectin in preventing vascular stenosis. The missing link of adipo-vascular axis. The Journal of biological chemistry 277, 37487-37491, doi:10.1074/jbc.M206083200 (2002). 
21 Piao, L. et al. Adiponectin/AdiopR1 signal inactivation contributes to impaired angiogenesis in mice of advanced age. International journal of cardiology 267, 150-155, doi:10.1016/j.ijcard.2018.05.089 (2018).

22 Iwabu, M. et al. Adiponectin and AdipoR1 regulate PGC-1alpha and mitochondria by $\mathrm{Ca}(2+)$ and AMPK/SIRT1. Nature 464, 1313-1319, doi:10.1038/nature08991 (2010).

23 Wang, X., Li, J., Tang, W., Zhao, Y. \& Li, X. Adiponectin receptor 1-mediated micro RNA-323-3p regulates functions of the MIN6 cell line via the AMPactivated protein kinase/sirtuin-1 pathway. The Journal of international medical research 46, 1693-1708, doi:10.1177/0300060518758584 (2018).

24 Potenza, M. A. et al. Activation of AMPK/SIRT1 axis is required for adiponectin-mediated preconditioning on myocardial ischemia-reperfusion (I/R) injury in rats. PloS one 14, e0210654, doi:10.1371/journal.pone.0210654 (2019).

25 Martinez-Huenchullan, S. F. et al. Skeletal muscle adiponectin induction in obesity and exercise. Metabolism: clinical and experimental 102, 154008, doi:10.1016/j.metabol.2019.154008 (2020).

26 Ding, M. et al. Vascular smooth muscle cell-derived adiponectin: a paracrine regulator of contractile phenotype. Journal of molecular and cellular cardiology 52, 474-484, doi:10.1016/j.yjmcc.2011.09.008 (2012).

27 Hsieh, C. J. \& Wang, P. W. Effect of cilostazol treatment on adiponectin and soluble CD40 ligand levels in diabetic patients with peripheral arterial occlusion disease. Circulation journal : official journal of the Japanese Circulation Society 73, 948-954, doi:10.1253/circj.cj-08-0905 (2009).

28 Shi, G. Y. et al. Plasmin and the regulation of tissue-type plasminogen activator biosynthesis in human endothelial cells. The Journal of biological chemistry 267, 19363-19368 (1992).

29 Percie du Sert, N. et al. Reporting animal research: Explanation and elaboration for the ARRIVE guidelines 2.0. PLoS biology 18, e3000411, doi:10.1371/journal.pbio.3000411 (2020).

30 Couffinhal, T. et al. Mouse model of angiogenesis. The American journal of pathology 152, 1667-1679 (1998).

31 Li, M., Qian, M., Kyler, K. \& Xu, J. Endothelial-Vascular Smooth Muscle Cells Interactions in Atherosclerosis. Frontiers in cardiovascular medicine 5, 151, doi:10.3389/fcvm.2018.00151 (2018).

32 Young, L. H., Li, J., Baron, S. J. \& Russell, R. R. AMP-activated protein kinase: a key stress signaling pathway in the heart. Trends in cardiovascular medicine 15, 110-118, doi:10.1016/j.tcm.2005.04.005 (2005). 
133 Chang, C. et al. AMPK-Dependent Phosphorylation of GAPDH Triggers Sirt1

$2 \quad$ Activation and Is Necessary for Autophagy upon Glucose Starvation.

3 Molecular cell 60, 930-940, doi:10.1016/j.molcel.2015.10.037 (2015).

$434 \mathrm{Hou}$, X. et al. SIRT1 regulates hepatocyte lipid metabolism through activating

5 AMP-activated protein kinase. The Journal of biological chemistry 283, 20015-

$6 \quad$ 20026, doi:10.1074/jbc.M802187200 (2008).

735 Yanai, H. \& Yoshida, H. Beneficial Effects of Adiponectin on Glucose and Lipid

$8 \quad$ Metabolism and Atherosclerotic Progression: Mechanisms and Perspectives.

9 International journal of molecular sciences 20, doi:10.3390/ijms20051190

10 (2019).

1136 Liu, C. et al. Adiponectin, TNF- $\alpha$ and inflammatory cytokines and risk of type 2

12 diabetes: A systematic review and meta-analysis. Cytokine 86, 100-109,

13 doi:10.1016/j.cyto.2016.06.028 (2016).

1437 Eslamian, M., Mohammadinejad, P., Aryan, Z., Nakhjavani, M. \& Esteghamati, 15 A. Positive Correlation of Serum Adiponectin with Lipid Profile in Patients with Type 2 Diabetes Mellitus is Affected by Metabolic Syndrome Status. Archives of Iranian medicine 19, 269-274 (2016).

1838 Hafiane, A., Gasbarrino, K. \& Daskalopoulou, S. S. The role of adiponectin in 19 cholesterol efflux and HDL biogenesis and metabolism. Metabolism: clinical and experimental 100, 153953, doi:10.1016/j.metabol.2019.153953 (2019). 
1 Figure 1.

A

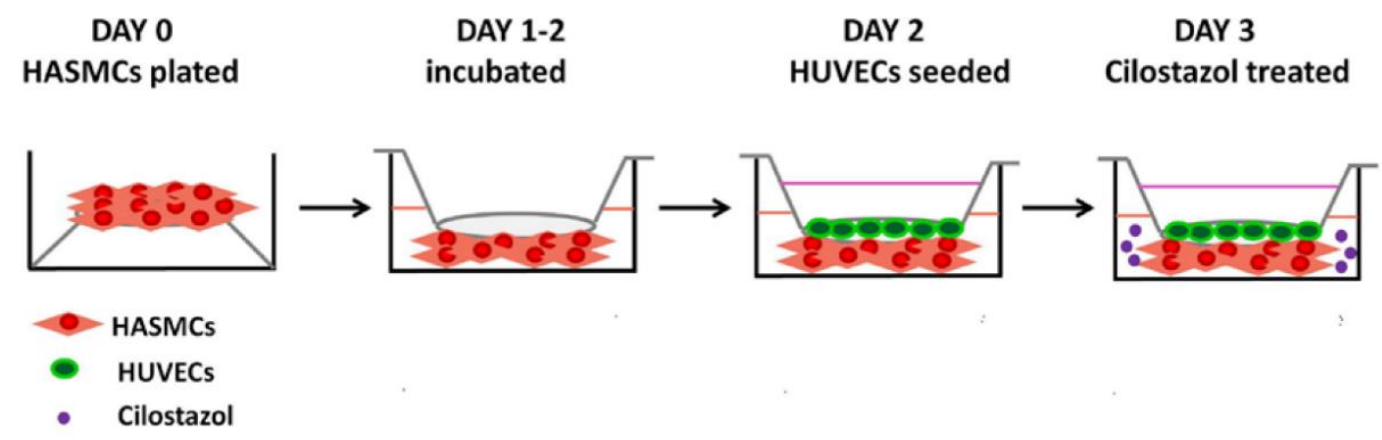

B
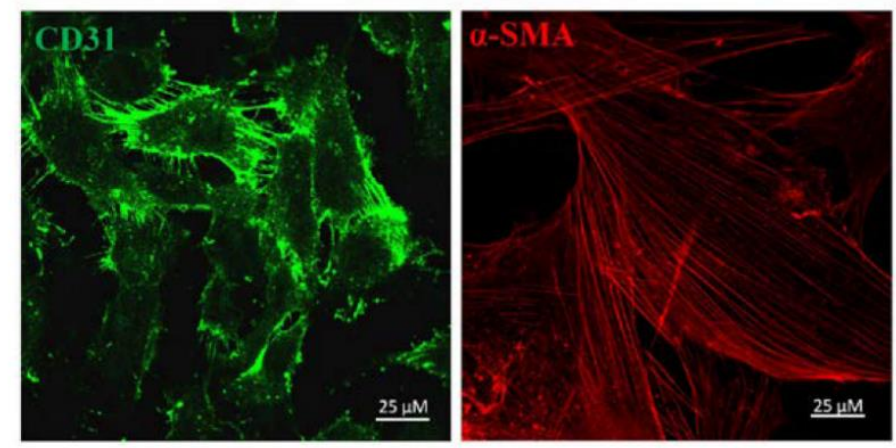

C

D
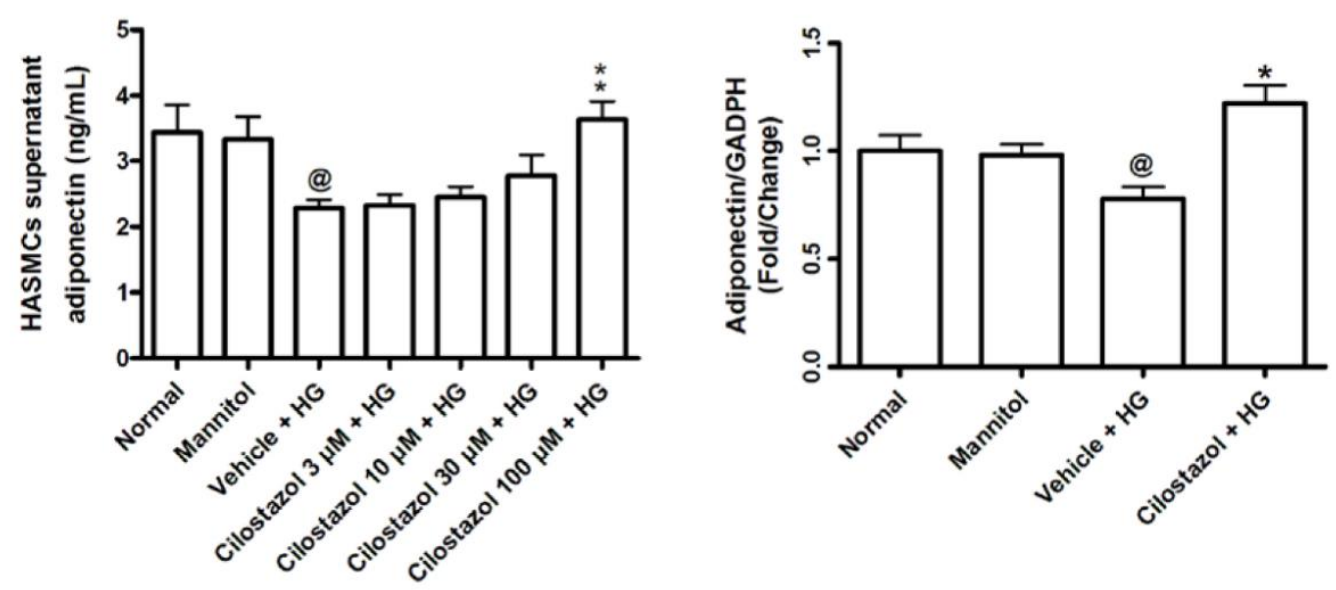

E

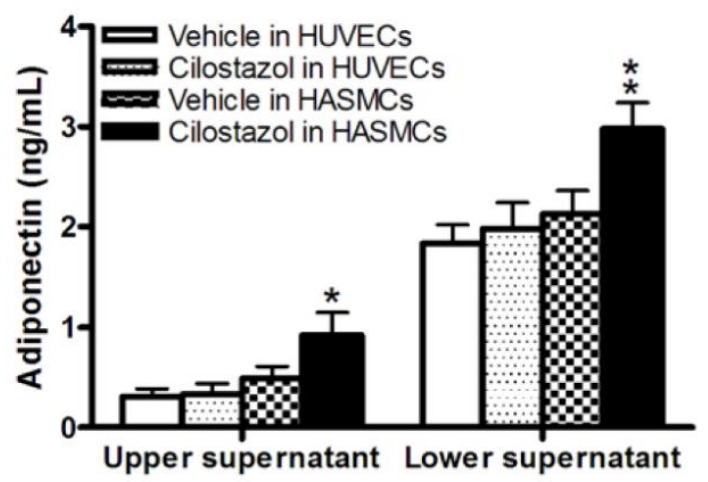


1 Figure 2.

A

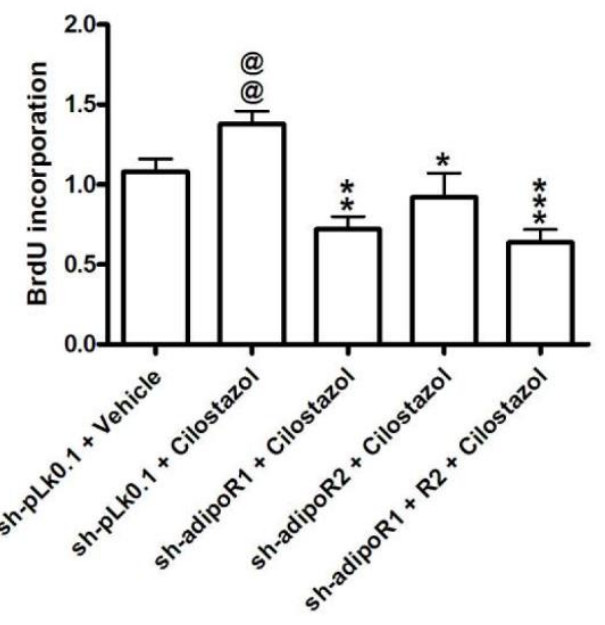

B

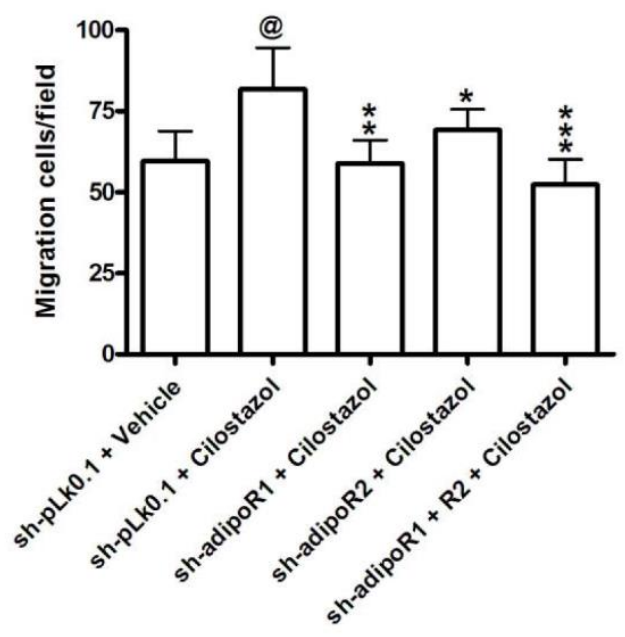

C
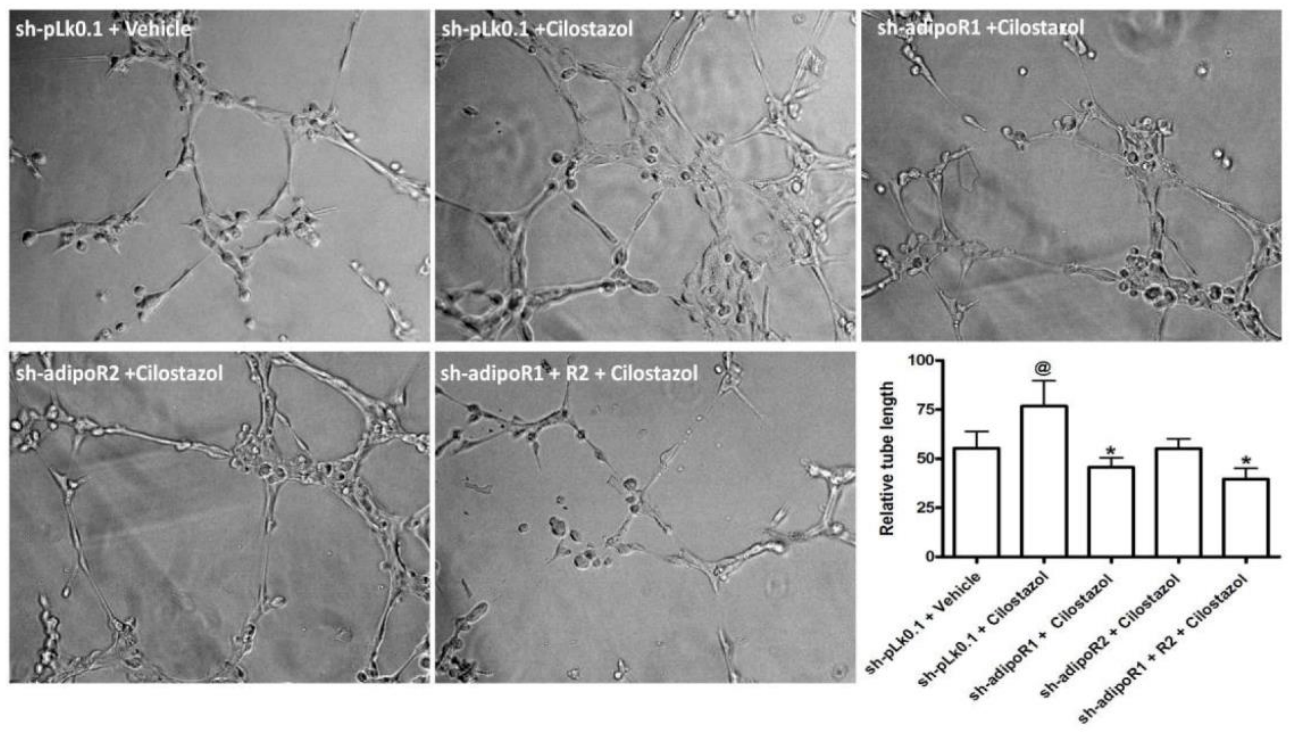

D

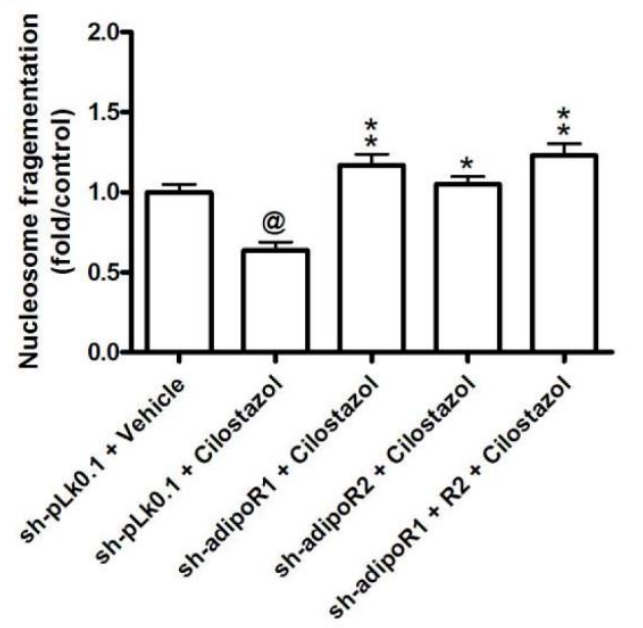

E

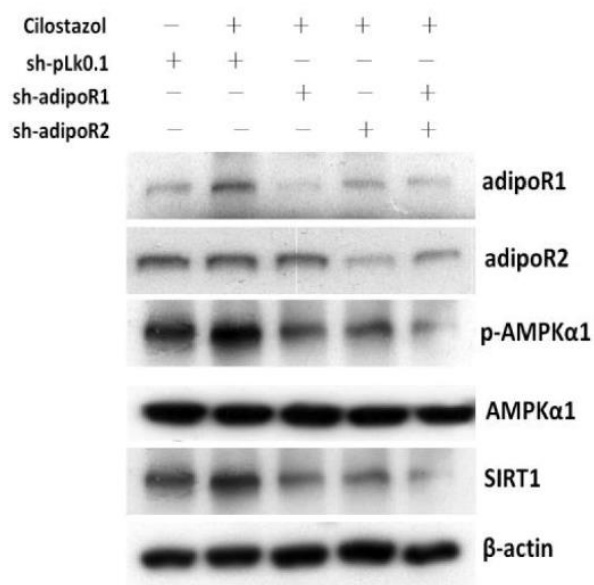


1 Figure 3.

A

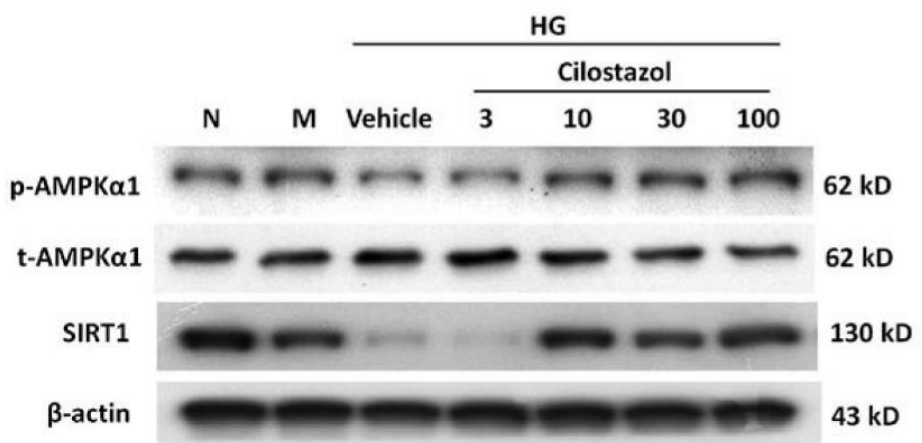

B

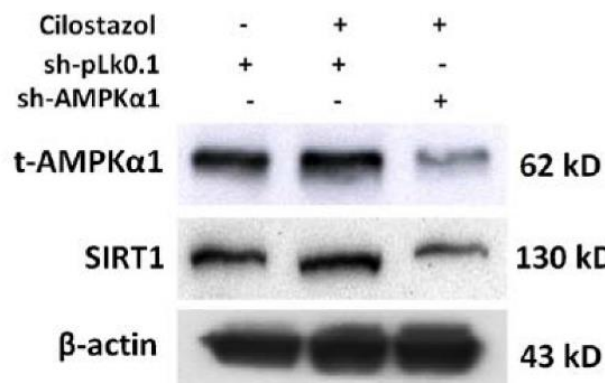

D

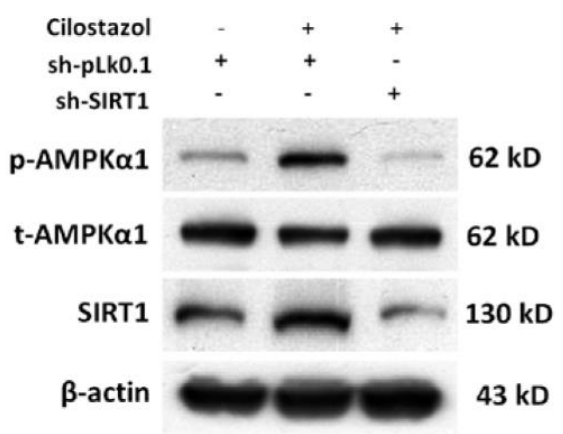

F

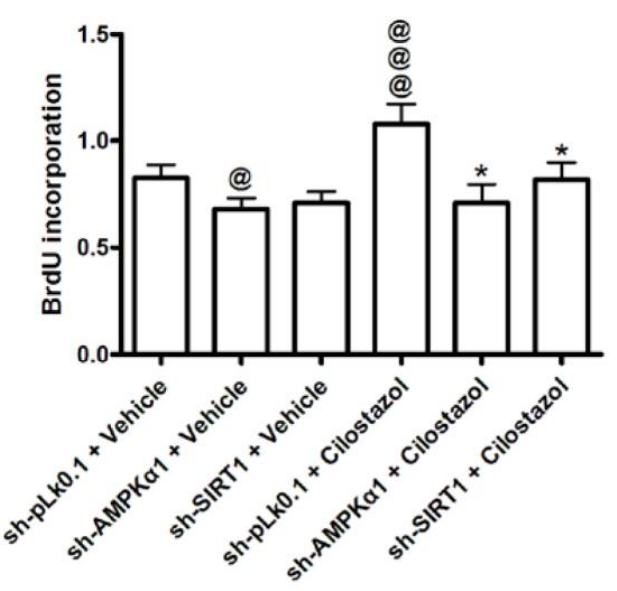

C

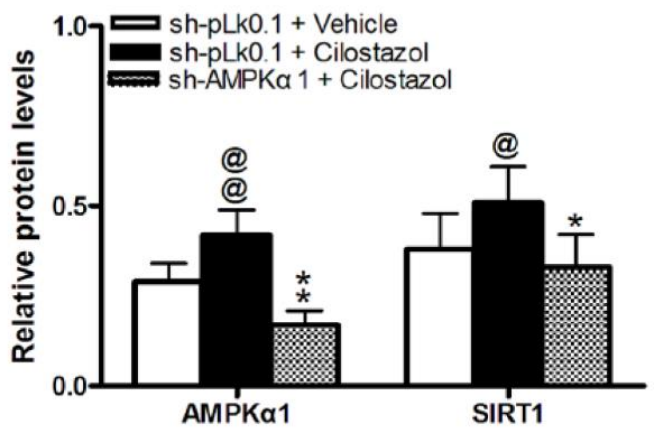

E

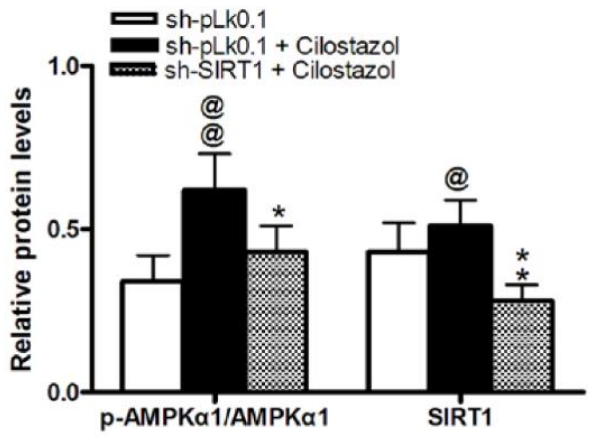

G

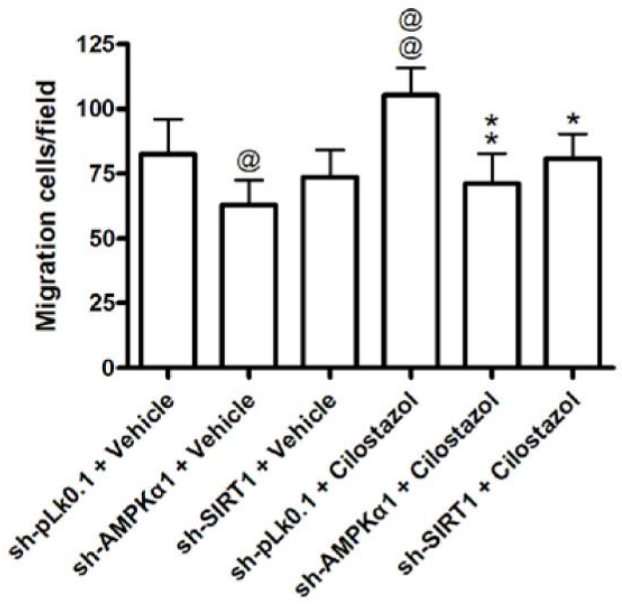


1 Figure 4.

A

B
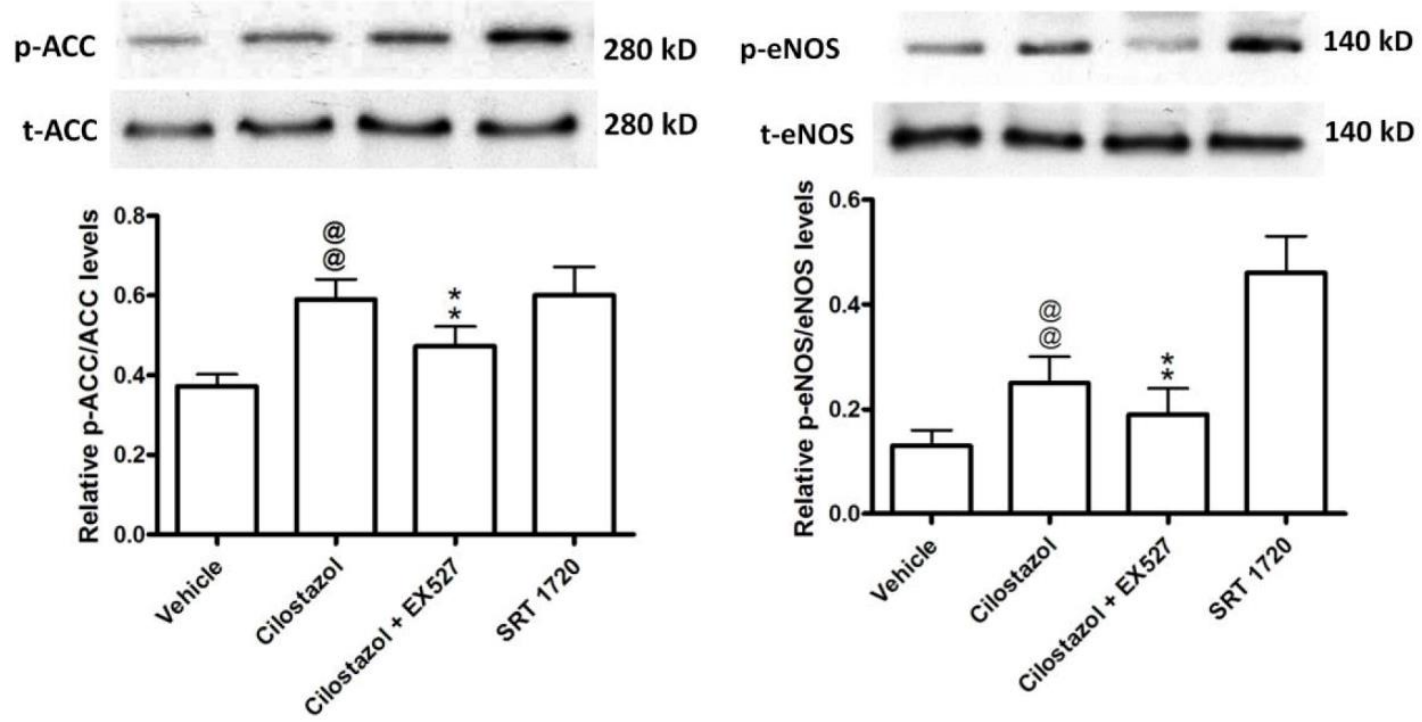

C

D
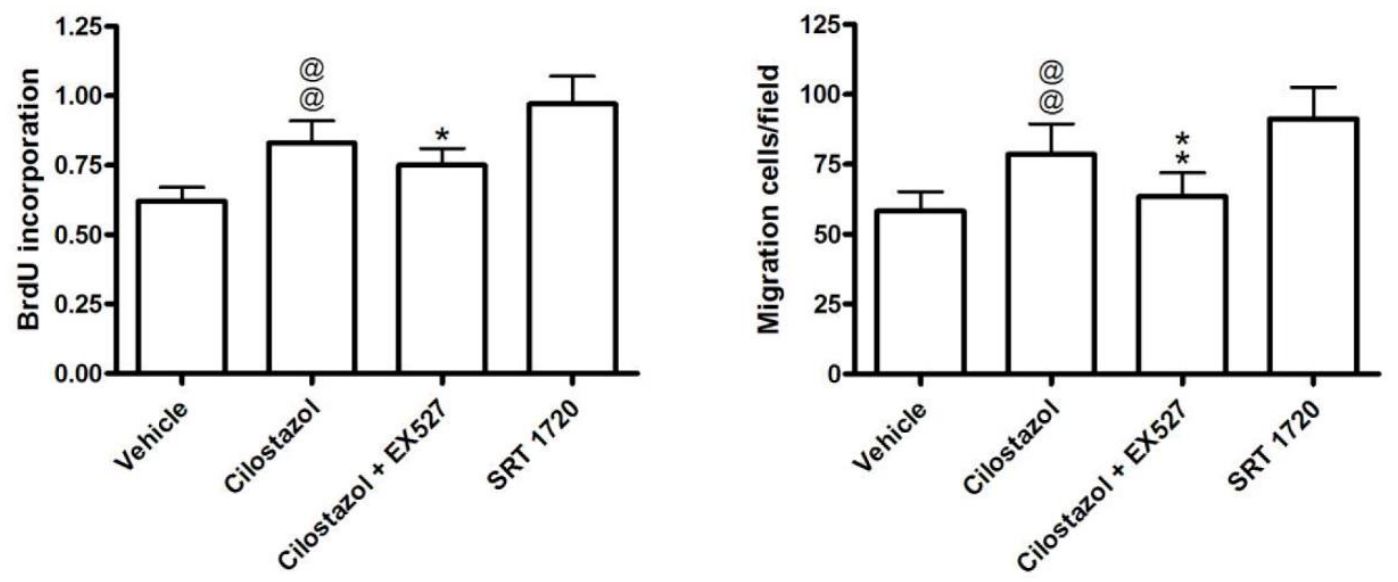

E

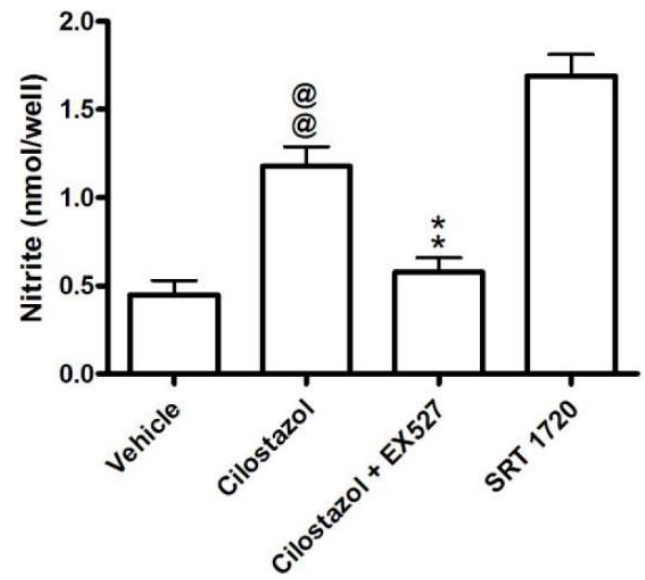


Figure 5.

A

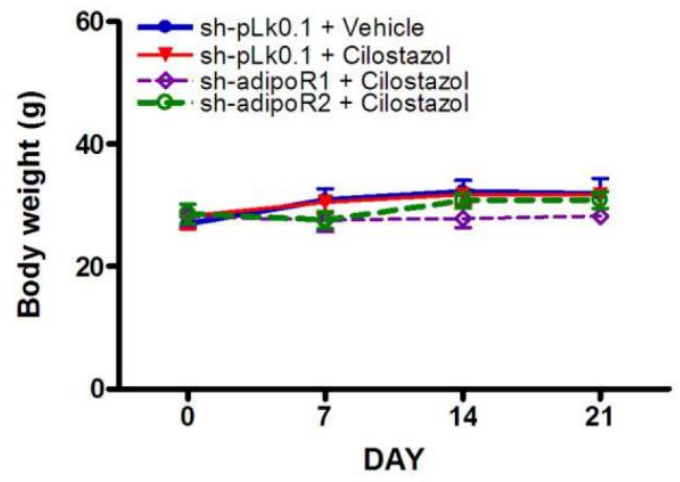

C

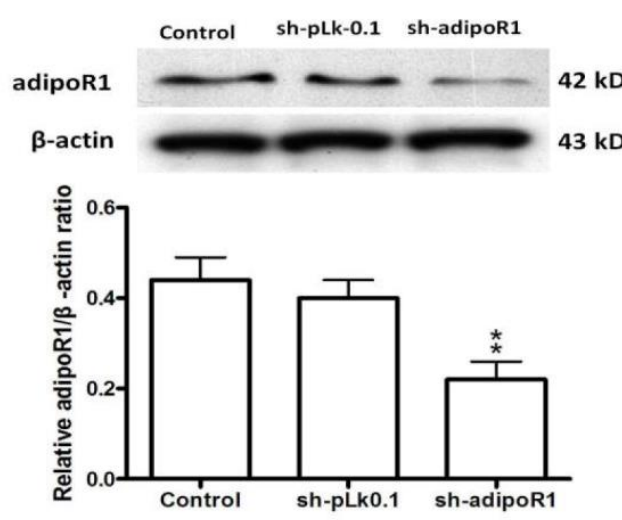

B

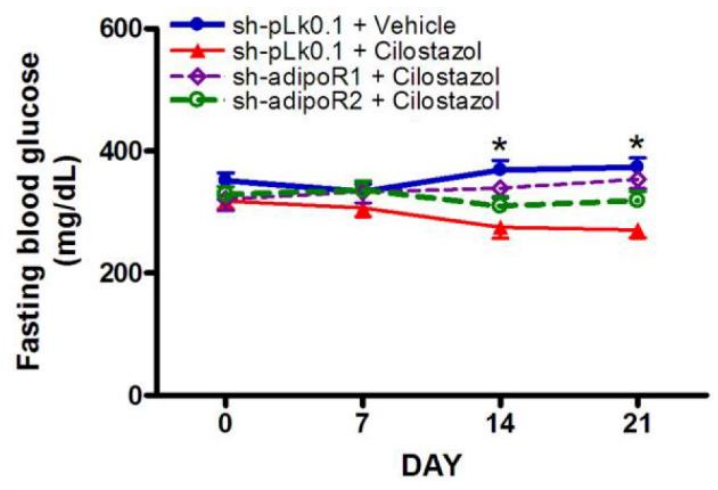

D

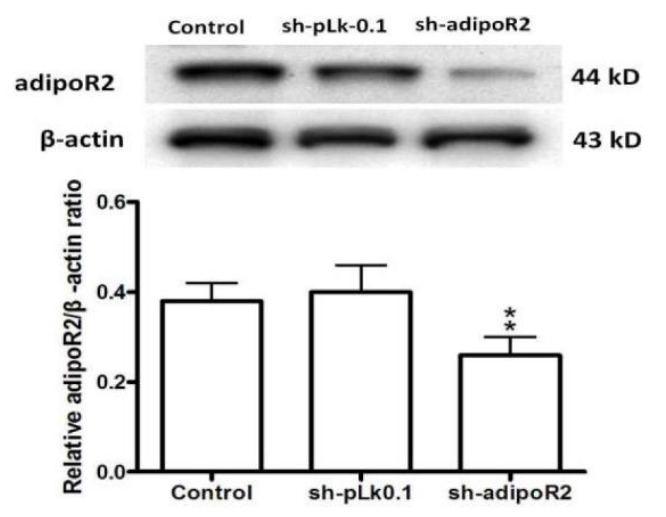

E

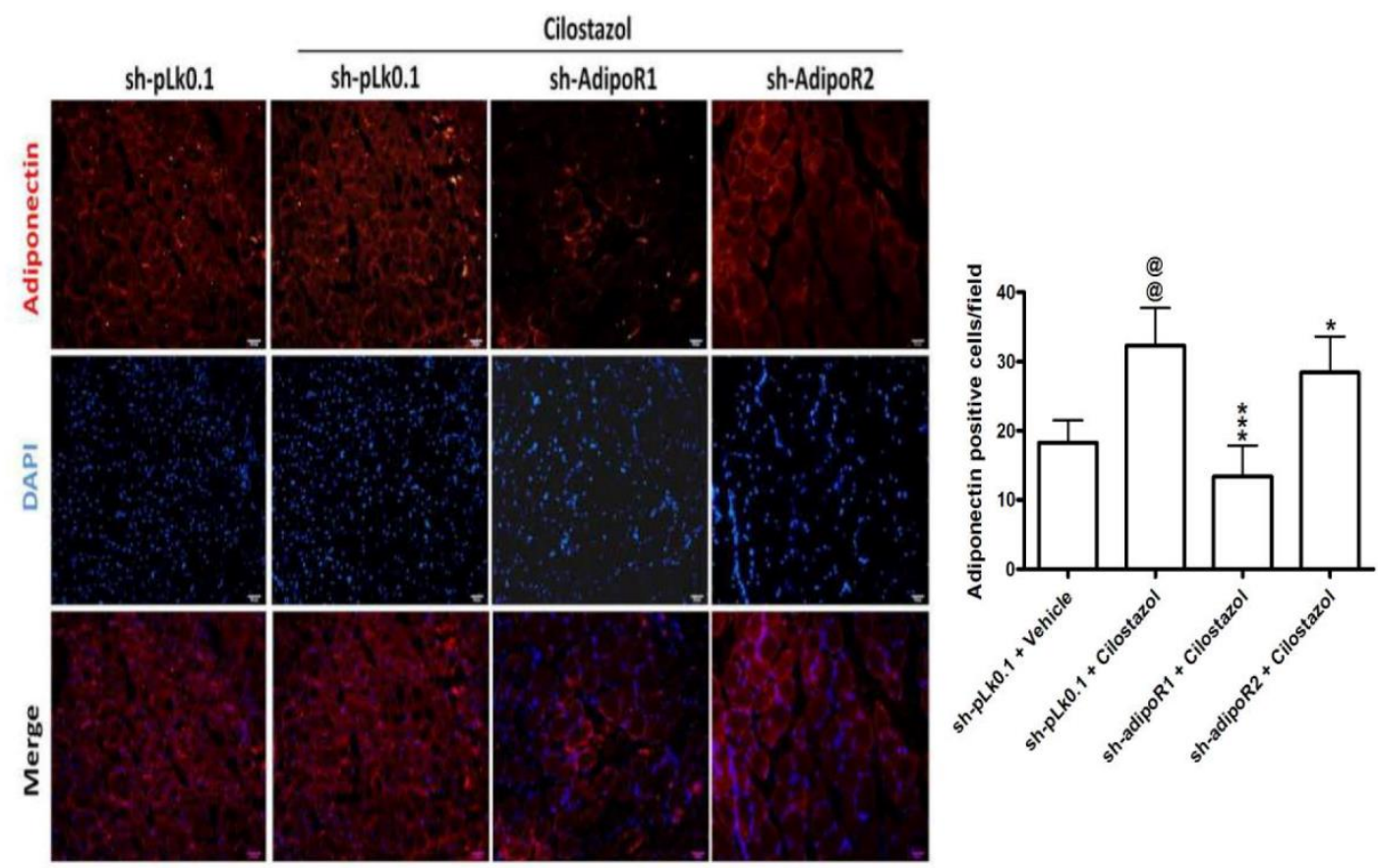


$1 \quad$ Figure 6.

A

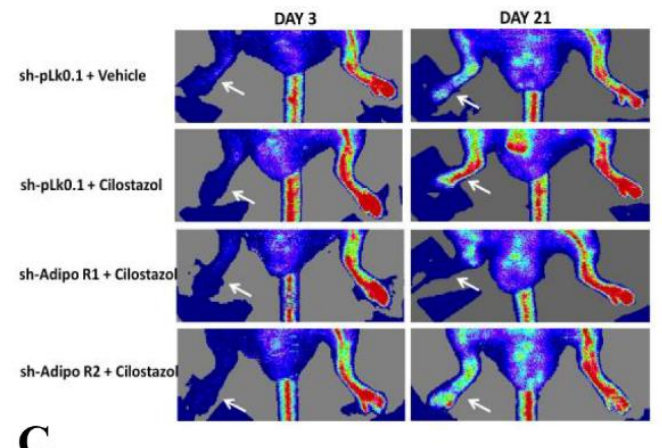

C

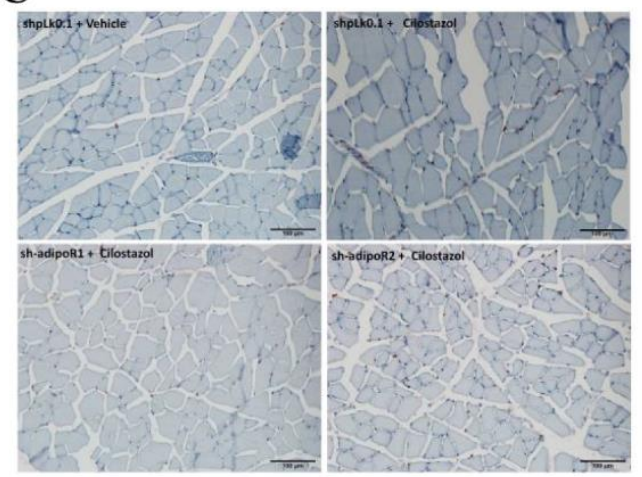

E

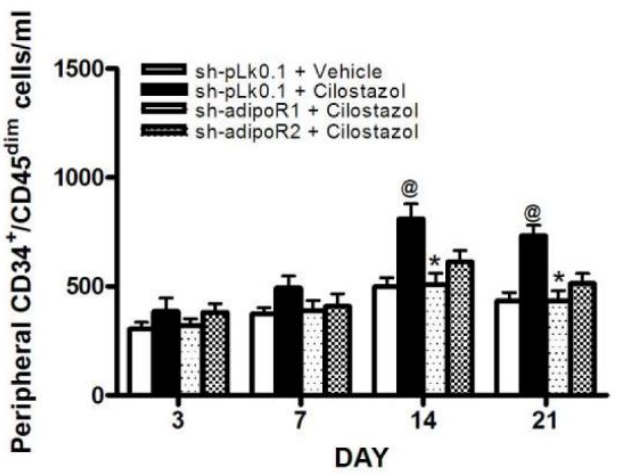

F

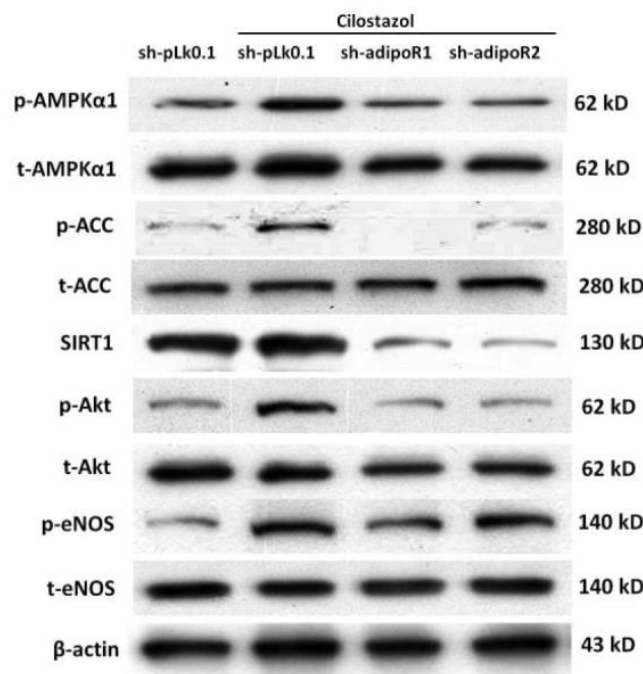

B

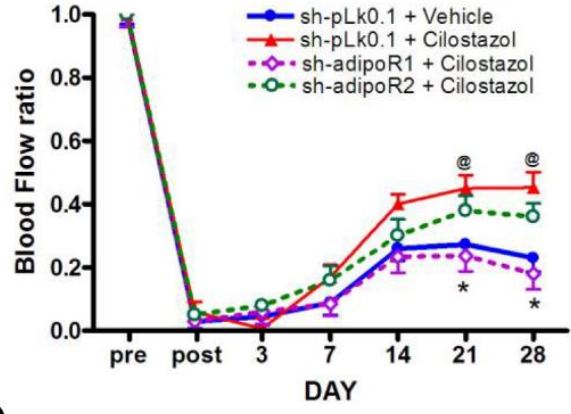

D

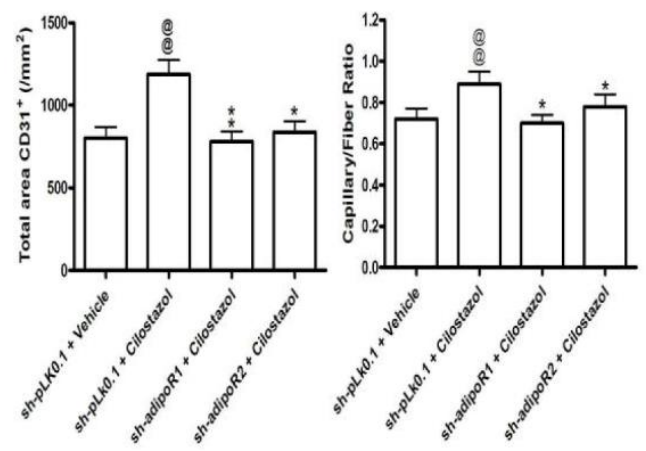

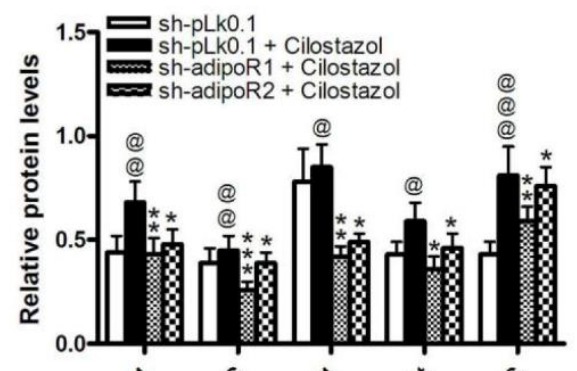

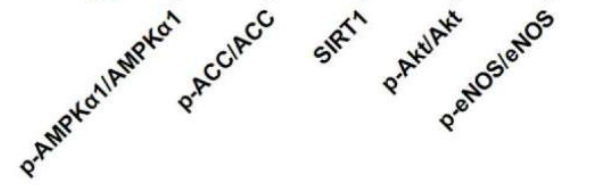


$1 \quad$ Figure 7.

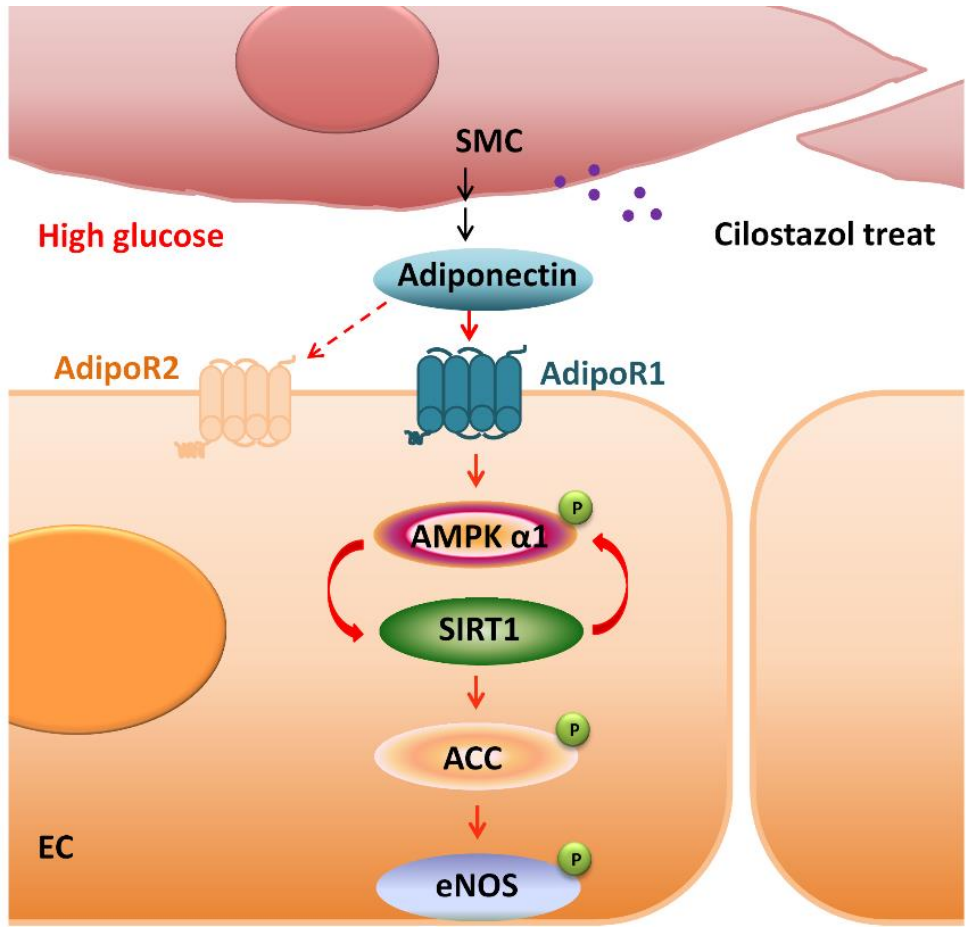




\section{Supplementary Files}

This is a list of supplementary files associated with this preprint. Click to download.

- Supplementarydata.doc 